\title{
Estudio a largo plazo (más de 10 años de evolución), de las complicaciones de las artroplastias trapecio-metacarpianas de tipo ARPE ${ }^{\circledR}$
}

\author{
M. A. Martín Ferrero ${ }^{\mathrm{I}}$, C. Simón Pérez ${ }^{\mathrm{I}}$, A. Mayo Iscar²; B. García Medrano ${ }^{\mathrm{I}}$, \\ J.J. Marcos Rodriguez ${ }^{\mathrm{I}}$, H. Hijazi Alsusi ${ }^{3}$ \\ IServicio de Cirugía Ortopédica y Traumatología del Hospital Clínico Universitario de Valladolid. España. \\ ${ }^{2}$ ProfesorTitular Departamento de Estadística Universidad de Valladolid. España \\ ${ }^{3}$ Servicio de Cirugía Ortopédica y Traumatología Complejo Hospitalario Rio Carrión de Palencia. España.
}

\begin{abstract}
Resumen: Objetivo: La artrosis trapecio-metacarpiana es una patología incapacitante, que produce dolor, disminución de la fuerza, pérdida de destreza en la pinza y limitación de la movilidad del pulgar. Cuando fracasa el tratamiento médico se pueden realizar diferentes técnicas quirúrgicas. Las prótesis totales de la articulación representan una de ellas. Desde mayo de 1999 hasta abril de 2004 se realizaron, de forma consecutiva, un total de II 6 prótesis totales de la articulación trapecio-metacarpiana en 103 pacientes que padecían artrosis, utilizando la prótesis no cementada y no constreñida tipo ARPE ${ }^{\circledast}$ (Biomet Spain Orthopaedics SL, Valencia). Se presentan en este estudio los resultados de la revisión clínica, funcional y radiológica de los pacientes revisados durante 10 años de evolución.

Material y Método: Los análisis de supervivencia fueron realizados mediante el método de Kaplan-Meier. De los 103 pacientes, 6 fueron excluidos y 97 pacientes, con 109 prótesis acabaron el estudio.

Resultados: En la revisión de los 10 años de evolución, 102 (93,6\%) eran prótesis funcionales y 7 (6,4\%) prótesis eran consideradas prótesis fallidas (3 retiradas por aflojamiento, 3 luxaciones y I subluxación y dolor). La probabilidad de supervivencia de Kaplan-Meier fue de $94.1 \%$ [CI95\% $(90.4 \%, 98.8 \%)]$. Radiográficamente, $88(86,3 \%)$ de las prótesis consideradas funcionales no mostraron evidencia de alteraciones del implante y 14 (13,7\%) mostraron cambios radiográficos que eran compatibles con la funcionalidad del implante. Se presenta en detalle el estudio de las complicaciones que han existido, con las posibles soluciones en las cirugias de revisión.

Conclusión: La prótesis total de ARPE ${ }^{\circledR}$ ofrece una alternativa de tratamiento segura y fiable en pacientes con artrosis trapecio-metacarpiana grado III y algunos de grado IV con buen capital óseo, sobre todo, en el trapecio. La mayoría de las complicaciones aparecen por defectos de indicación o por defectos de la técnica quirúrgica y se pueden resolver con recambio protésico o con trapecectomía dependiendo de las circunstancias del implante y del paciente.
\end{abstract}

Palabras claves: Artroplastias no cementadas, rizartrosis, prótesis trapecio-metacarpianas, artroplastias carpo-metacarpianas del pulgar.

\section{Long term review (more than 10 years) of complications with ARPE ${ }^{\circledR}$ type total joint trapeziometacarpal arthroplasty}

Abstract: Objetive:The trapezio-metacarpal arthritis is a disabling disease that causes pain, decreased strength, loss of ability in the clamp and limited movement of the thumb. When medical treatment fails surgery can be performed in multiple ways. One of those may be prosthesis. From May 1999 to April 2004 were performed, consecutively, a total of 116 total prosthesis of the trapezio-metacarpal joint in 103 patients suffering from osteoarthritis, using uncemented and unconstrained ARPE ${ }^{\circledast}$ (Biomet Spain Orthopaedics SL, Valencia) prosthesis. Clinical, functional and radiological results at 10-year follow-up are presented. Survival analysis was performed using the Kaplan-Meier method.

Methods: Of the 103 patients, 6 were excluded, remaining 97 patients with 109 prostheses that completed the study.

Results: In a review of 10 years of evolution, $102(93.6 \%)$ were functional prosthesis and 7 (6.4\%) were considered failed prosthesis prostheses $(3$ removed by loosening of the cup, 3 were dislocated, and I with subluxation and painful). Survival estimate for functional implants over 10 years was $94.1 \%$ [95\% Cl $(90.4 \%, 98.8 \%)]$. Radiographically, $88(86.3 \%)$ of the considered functional prostheses showed no evidence of alterations of the implant, and 14 (13.7\%) showed radiographic changes that were compatible with the functionality of the implant. Complications are extensively presented in this study, with the possible solutions with revision surgeries.

Conclusion:Total ARPE ${ }^{\circledR}$ prosthesis offers a safe and reliable alternative treatment in patients with trapezio-metacarpal osteoarthritis grade III and grade IV with some good bone stock, especially in the trapezius. Most complications occur due to indication defects or to defects of the surgical technique and can be solved with prosthetic replacement or trapeziectomy depending on the circumstances of the implant and of the patient.

Key words: Uncemented arthroplasties, osteoarthritis of the thumb, trapezium-metacarpal prosthesis, thumb carpometacarpal joint arthroplasty.

La artrosis trapecio-metacarpiana es una patología muy incapacitante, ya que produce dolor a la movilización del pulgar, disminución de la fuerza, pérdida de destreza en la pinza, limitación de la movilidad y, dejada a su evolución espontánea, deformidad progresiva de la columna del pulgar por aducción del
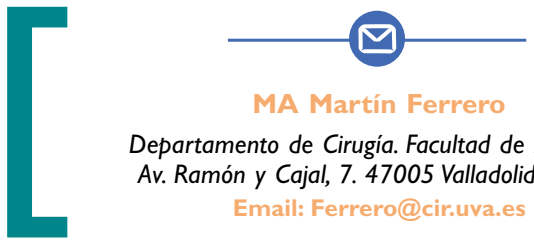

Departamento de Cirugía. Facultad de Medicina, Av. Ramón y Cajal, 7. 47005 Valladolid España Email: Ferrero@cir.uva.es 
primer dedo, subluxación radial de la base del primer metacarpiano y la consiguiente hiperextensión de la articulación metacarpofalangica (MF)'.

Cuando fracasa el tratamiento médico, existen numerosas técnicas quirúrgicas; la elección de una u otra técnica viene determinada, entre otros factores, por la edad del paciente y la funcionalidad que debe ejercer su mano en sus actividades diarias, el estadio radiográfico de la enfermedad y la experiencia del cirujano sobre cada técnica en particular 2,3.

La trapecectomía sigue siendo el procedimiento más frecuente para el tratamiento de la artrosis trapeciometacarpiana $y$, aunque se han publicado resultados adversos ${ }^{4}$, todavía continúa siendo el procedimiento más fiable ${ }^{5,6}$.

Desde que De la Caffinière ${ }^{7}$ describió la primera prótesis total, en 1973, la artroplastia se ha convertido en uno de los tratamientos para este tipo de patología, se han diseñado muchos tipos de prótesis, incluyendo espaciadores ${ }^{8,9}$, hemiartroplastias ${ }^{10,11}$ y prótesis totales ${ }^{12-14}$, con los que se han obtenido resultados favorables ${ }^{1,3,15-20}$; concretamente, los pacientes se recuperan en menos tiempo que con la trapecectomía y presentan mejor funcionalidad y más fuerza de la pinza ${ }^{\mid 4,18,19,21}$. Sin embargo, las prótesis plantean algunos problemas: en las prótesis cementadas existe un porcentaje elevado de aflojamiento de los componentes y en las no cementadas hay múltiples publicaciones con resultados desfavorables $1,8,1,1,1,18,20,22,23$. Los fallos se han relacionado, en estas prótesis no cementadas, con los cotilos roscados, en los que ha habido fallos de hasta el 47\% a los 6 años de evolución. Resultados similares habían sido ya publicados hace unos años con los cotilos roscados en las prótesis de cadera ${ }^{24}$.

La artroplastia de ARPE ${ }^{\circledR}$ (Biomet Spain Orthopaedics $S L$, Valencia) fue presentada en $\left.199\right|^{12}$. Se trata de una artroplastia modular de tipo esferoidal, no cementada (recubierta de hidroxiapatita) y no constreñida, con el cotilo insertado en el trapecio y el vástago en el metacarpiano del pulgar.

El objetivo de este estudio es comentar resumidamente los resultados a largo plazo (más de 10 años) de 116 artroplastias de ARPE ${ }^{\circledR}$ consecutivas colocadas en nuestro servicio $y$, sobre todo, el estudio de las complicaciones que han existido y su posible solución.

\section{MATERIALY MÉTODO}

Se realiza un estudio prospectivo en la Unidad de mano del Hospital Universitario de Valladolid de todos los pacientes intervenidos de artrosis trapecio-metacarpiana del pulgar mediante artroplastia total de tipo ARPE $^{\circledR}$, desde mayo de 1999 a abril de 2004. Se han recogido los datos relativos al paciente, a la cirugía y al implante.

Inicialmente, 103 pacientes consecutivos (7 prótesis) entraron en el estudio. Seis de ellos, no acabaron el seguimiento: 4 por estar fallecidos en la revisión de los 10 años y 2 por enfermedad de Alzheimer. Por tanto el estudio se ha realizado en 97 pacientes, con 109 artroplastias. Noventa y siete pulgares presentaban artrosis de grado III de Eaton y 12 de grado IV. Noventa y cuatro pacientes (I06 artroplastias) eran mujeres y 3 hombres. La edad media era de 58,8 años (rango 45-75 años). El tiempo medio de seguimiento fue de 10,5 años (rango 9,8- | 3,3 años). Sesenta (55\%) pulgares eran derechos y el resto izquierdos. Doce ( $11 \%$ ) fueron bilaterales. Los cotilos del número 9 y los vástagos del numero 8 son los más frecuentes. Todos los cuellos, excepto 2 , fueron inclinados. Sesenta y dos $(56,9 \%)$ de las prótesis acudieron a rehabilitación y el resto hicieron un protocolo de ejercicios que les proporcionamos.

Ciento cuatro artroplastias $(95,4 \%)$ completaron la revisión clínica y radiológica presencial y 5 (4,6\%) fueron revisadas telefónicamente y no se dispuso de radiografías.

\section{Técnica quirúrrgica}

Se comenzó realizando la técnica quirúrgica como la describió el diseñador de la prótesis ${ }^{12}$, por vía anteroexterna, con osteotomía del trapecio y del metacarpiano, pero se encontraron varias dificultades técnicas que influían en la evolución posterior del implante, por ello se varió la técnica a la que se describe a continuación.

Un único cirujano (M-F) realizó todas las artroplastias por la vía estándar dorso-radial por una incisión de unos $4 \mathrm{~cm}$. de longitud, entre los tendones del Extensor Pollicis Brevis y el Abductor Pollicis Longus (APL). Se abre longitudinalmente la cápsula dorsal de la articulación trapecio-metacarpiana (TMC) y después de liberar los extremos articulares y retirar los osteofitos se realiza una osteotomía ligeramente oblicua en sentido palmar, con una sierra frontal, para resecar los 6-8 $\mathrm{mm}$ proximales del primer metacarpiano (2) Figura I. 

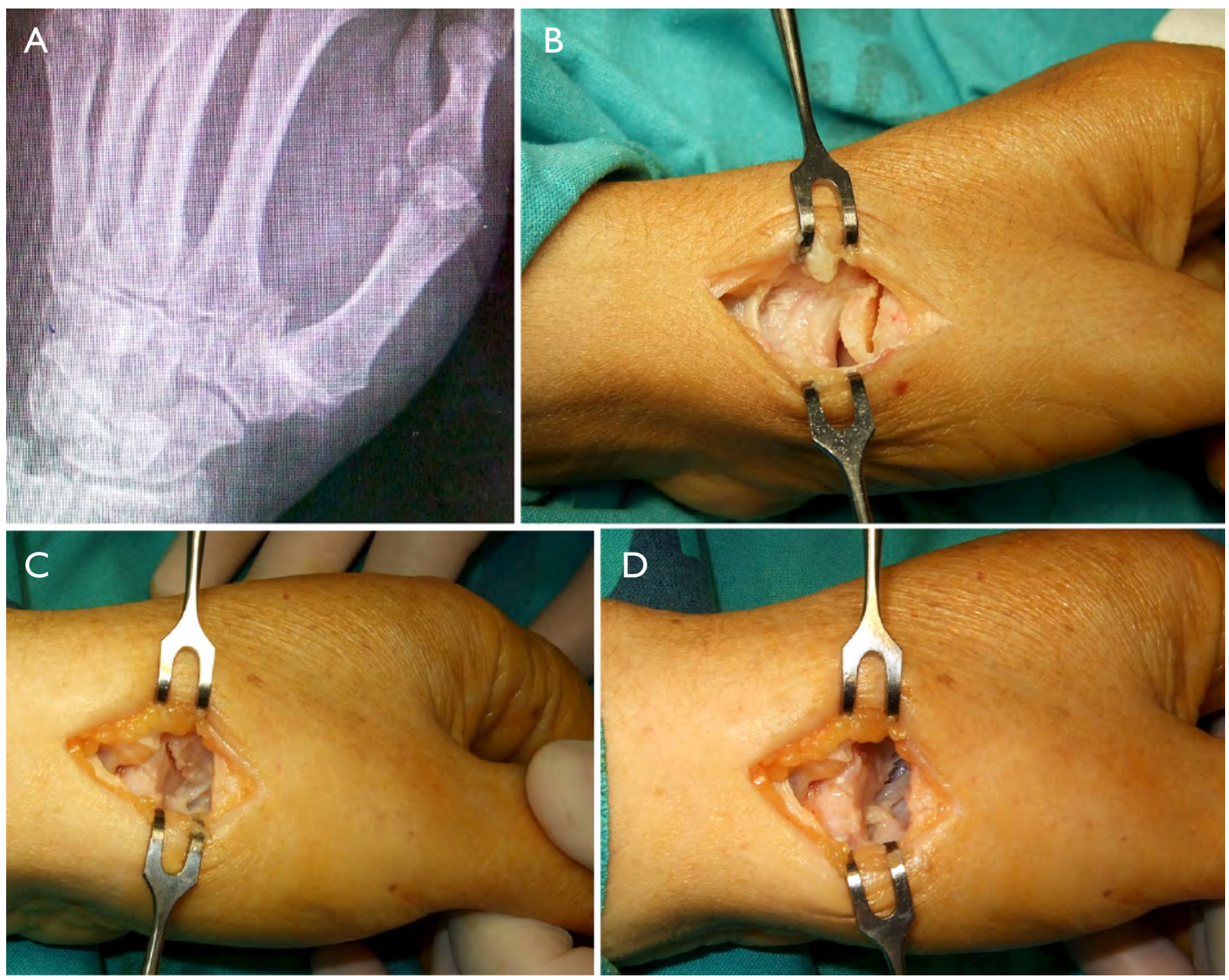

Figura $\|$. Técnica quirúrgica: A. RX preoperatorio mostrando osteofito en el lado cubital, B. incisión mostrando la resección de la base del metacarpiano, C. osteotomía de resección del osteofito cubital y D. amplio espacio que queda para poder colocar el implante.

Esto permite separar el metacarpiano y exponer perfectamente la superficie articular distal del trapecio, donde se calcula con facilidad el centro geométrico. En este punto se perfora un orificio con un punzón pequeño, el orificio creado se amplía con cucharillas de legrado de diferente tamaño hasta llegar a tener un orificio que aloje la fresa del cotilo del número 9 provista por la casa comercial. Con ella se acaba de realizar el orificio donde irá encajado el cotilo protésico. No se usan fresas motorizadas. Con el mismo punzón se abre el canal intramedular del metacarpiano, que se va ampliando con las raspas provistas por la casa comercial, hasta el tamaño adecuado. Se coloca el cotilo protésico adecuado "press fit", con los bordes apoyados sobre el hueso subcondral para incrementar la estabilidad primaria del implante. Posteriormente, se introduce el vástago protésico con la parte dorsal, coincidiendo en un plano paralelo a la uña del primer dedo. Se coloca la cabeza-cuello de prueba y se procede a la reducción de la prótesis. En este momento se realiza un test de estabilidad (2) Figura 2 y se buscan las posibles zonas de roce de los extremos óseos en todas las posiciones $y$, ante cualquier sospecha de inestabilidad, se realiza un estudio de radiología intraoperatorio para corregir las deficiencias. La longitud exacta del cuello se calcula de la siguiente manera: con el pulgar en retroposición completa, el pliegue palmar de la mano y el de la articulación interfalángica del pulgar deben estar a la misma altura (1) Figura 3. Se coloca entonces la cabeza-cuello definitivo, se reduce la prótesis y se vuelve a probar la estabilidad. Se realiza el cierre capsular y el de la piel con suturas reabsorbibles. Se coloca una inmovilización del pulgar en posición funcional con una férula de yeso corta, durante tres semanas, para evitar la posibilidad de luxación. A las tres semanas se retira la férula y el paciente comienza una tanda de ejercicios protocolizada. 

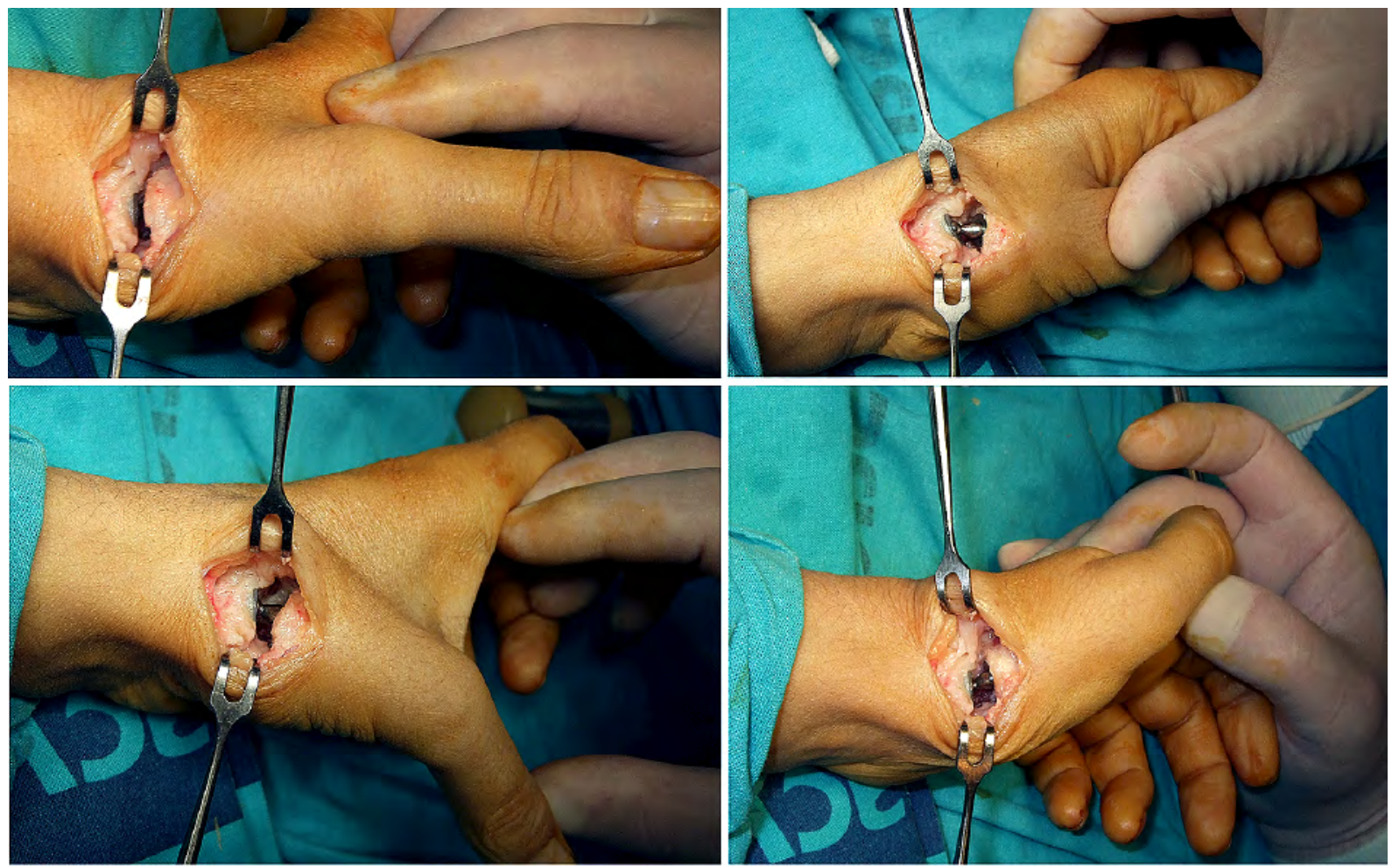

Figura. 2. Técnica quirúrgica: estrés en todas las direcciones al que se somete al implante antes de cerrar para probar su estabilidad.

\section{Evaluación de los pacientes}

Se realizaron estudios clínicos y radiográficos preoperatorios, a los tres meses, a los 5 años y a los 10 años postoperatorios.

El examen clínico consistió en el análisis de: a) movilidad, siguiendo los criterios de "International Federation of Societies for Surgery of the Hand"25, que considera la posición de descanso del pulgar en $30^{\circ}$ de abducción palmar y $10^{\circ}$ de extensión (abducción radial), y oposición usando los criterios de Kapandji26, y b) fuerza de la pinza del pulpejo del pulgar al lateral del dedo índice, se midió mediante el "B\&L Engineering mechanical pinch gauge".

El examen radiológico se realizó mediante estudio anteroposterior y oblicuo de la mano. Las radiografías preoperatorias fueron clasificadas según los criterios de Eaton $^{27}$, y la radiografías postoperatorias fueron evaluadas con respecto al alineamiento de los componentes del implante y al aflojamiento.

El dolor fue medido usando la escala visual analógica (EVA) y la satisfacción del paciente, mediante el cuestionario DASH (disabilities of the arm, shoulder, and hand) validado en idioma Castellano ${ }^{28}$.
Para la evaluación de resultados se definieron dos tipos de prótesis: prótesis funcionales y prótesis fallidas o fracasadas.

Las prótesis funcionales fueron aquellas que permiten realizar al paciente los movimientos y tareas de la mano cómodamente, sin dolor o con dolor leve ocasional a esfuerzos, sujetar objetos, cortar, pinzar llaves, coger latas, botellas, bolsas, etc. Y no muestran ninguno de los signos mayores de fracaso radiológico. Cuando una prótesis ha fallado, por ejemplo, por luxación, pero se ha revisado quirúrgicamente y se consi-

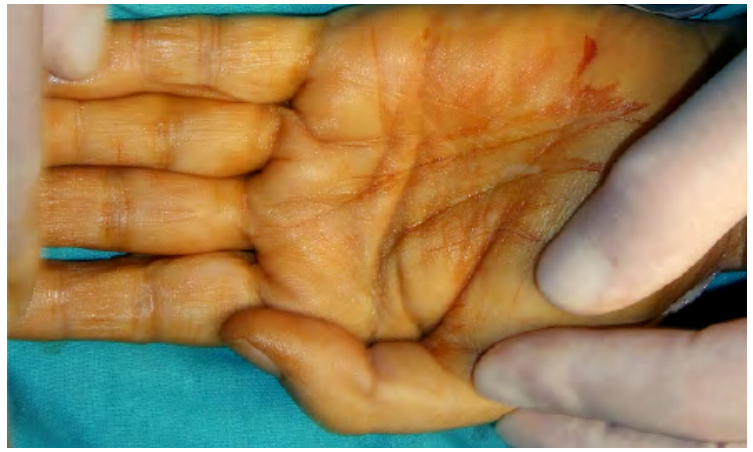

Figura 3. Modo de calcular la longitud del cuello protésico idóneo (explicación en el texto). 

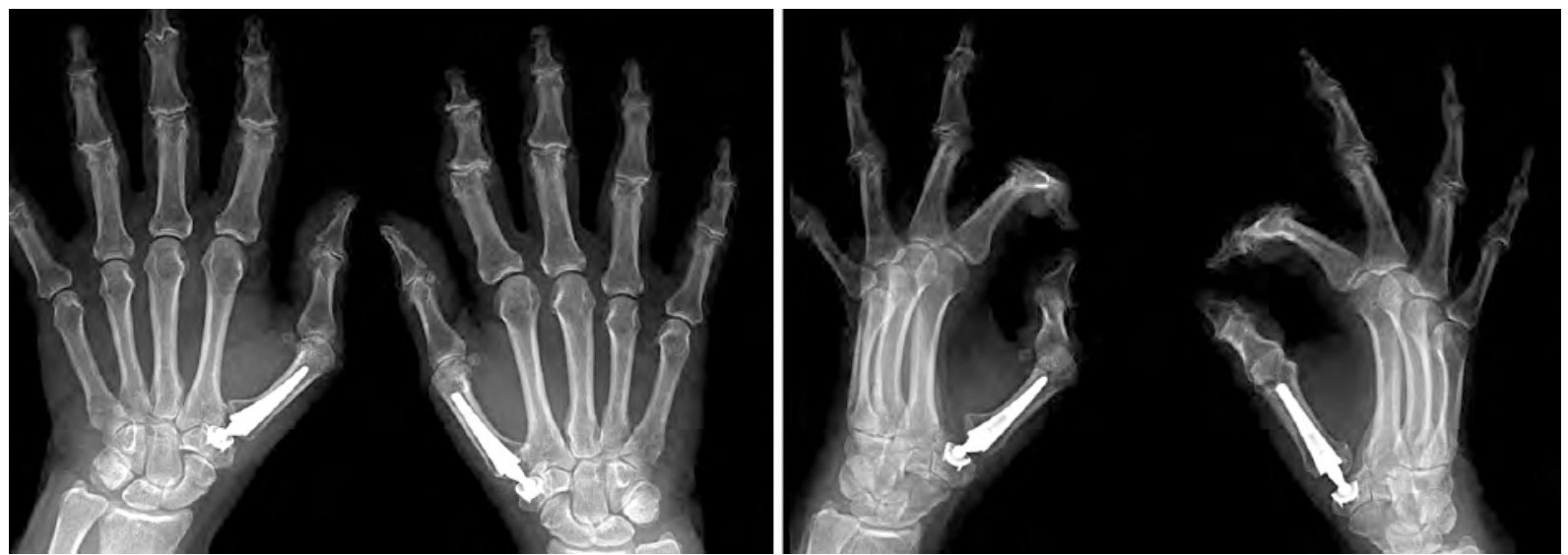

Figura 4. Paciente de 73 años en la revisión final, con prótesis de ARPE ${ }^{\circledR}$ (Biomet, Warsaw, Indiana) bilateral (núm. 42 y 43). Lado derecho: doce años de evolución. Lado izquierdo: 10 años de evolución. Buena funcionalidad de ambos pulgares, restringida levemente por la artrosis en el resto de la mano.

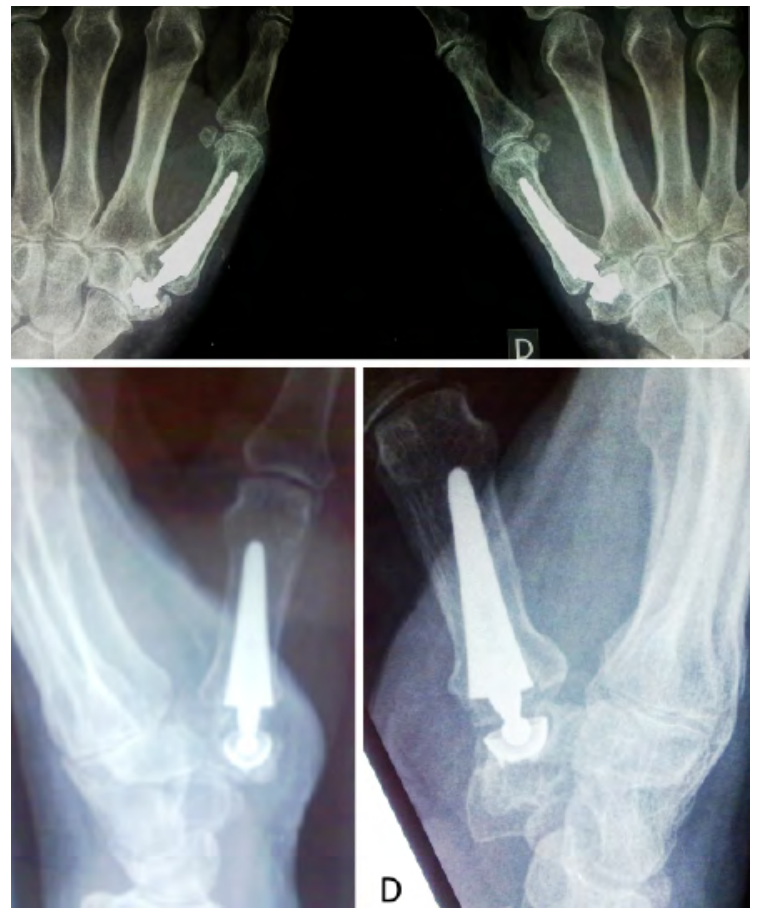

Figura 5. Paciente de 72 años a la revisión final, con prótesis bilateral: aflojamiento protésico asintomático en el lado izquierdo (núm. 63) a los 7 años de evolución, RX en la revisión de los 10 años. Obsérvese que a pesar de llevar 3 años con aflojamiento la prótesis el cotilo sigue en buena posición.

gue un buen resultado clínico en la revisión de los 10 años, se la considera funcional (2) Figura 4.

Prótesis fallidas son aquellas, que en el momento de la revisión, tienen un criterio mayor o al menos 2 criterios menores de fracaso:

\section{- Criterios mayores:}

1. Luxación de los componentes

2. Dolor EVA $\geq 5$

3. $\mathrm{DASH} \geq 40$

\section{- Criterios menores:}

I. Aflojamiento del cotilo sin cambios de posicionamiento a lo largo del tiempo. (2) Figura 5

2. Mal posición de los componentes con subluxación

3. EVA de 4

4. DASH de 30 a 40

\section{Análisis estadístico}

La variables continuas han sido evaluadas mediante la media y la desviación estándar (SD) o mediante la media y rango de datos. El test de Chi-cuadrado se utilizó para comparar frecuencias entre subgrupos de pacientes. Los métodos no paramétricos fueron usados en algunas ocasiones ${ }^{29}$. El método de Kaplan-Meier se utilizó para estimar la probabilidad de supervivencia a lo largo del tiempo ${ }^{30}$. El análisis estadístico y la determinación de la significación estadística $(P<0.05)$ se realizó con la ayuda del paquete SPSS (SPSS Inc., Chicago, IL).

El estudio fue aprobado por el comité ético del hospital. Los pacientes dieron su consentimiento.

Resultados: En la revisión de los 10 años de evolución, 102 (93,6\%) eran prótesis funcionales y 7 prótesis (6,4\%) eran consideradas prótesis fallidas (3 retiradas por aflojamiento del cotilo, 3 luxaciones y I subluxación importante y dolor).

La probabilidad de supervivencia a 10 años de Kaplan-Meier fue de 94.1\% [Cl95\% (90.4\%,98.8\%)] 
(1) Figura 6. No existe diferencia con significación estadística entre el grupo III y el grupo IV de Eaton.

Los datos generales de los resultados de las prótesis son los siguientes:

En la (1) Figura 7 se puede observar el dolor en la escala EVA preoperatorio y a los 10 años de evolución. Los valores preoperatorios se encuentran todos por encima de 6 y los postoperatorios, en su gran mayoría, por debajo de 4 ( $p<0,00$ I). Noventa y tres pulgares $(85,3 \%)$ no presentan dolor o dolor leve solamente con tareas muy intensas, 10 (9,2\%) tenían malestar con los ejercicios y $6(5,5 \%)$ padecían dolor en reposo.

En la (2) Figura 8 se muestran los valores medios del DASH a los 5 años, que era 18.3 (rango 5.6-36.8) y a los 10 años de evolución era 20.1 (rango 6.7-39.8) $(p=0.62)$. No disponemos del DASH preoperatorio. Observamos que los datos del DASH varían poco entre los 5 y los 10 años de evolución, lo que supone que las prótesis mantienen la funcionalidad.

Ciento cuatro paciente $(95,4 \%)$ refirieron que se encontraban satisfechos con la apariencia estética del pulgar operado.

En la (2) Tabla I queda reflejada la movilidad y el grado de oposición preoperatoria y postoperatoria a los 10 años. Se observa buena movilidad que presentan las prótesis a los 10 años de evolución, con un aumento significativo en todos los movimientos con respecto al preoperatorio $(\mathrm{p}<0,00 \mathrm{I})$.

La media de la fuerza de la pinza se incrementó desde $4.3 \mathrm{~kg}$ (SD 2.3) preoperatoria a $5.6 \mathrm{~kg}(\mathrm{SD} 2.6)$ a los 10 años de evolución ( $p=0.005$ ).

Ochenta y ocho (86,3\%) de las prótesis funcionales no mostraron evidencia de alteraciones radiográficas del implante y en catorce $(13,7 \%)$ se han observado ciertas alteraciones radiológicas que son compatibles con la funcionalidad de la prótesis. Estos cambios incluyen: (a) una ligera malposición de los componentes que estuvo presente desde la implantación inicial y no cambian con el tiempo, en once pacientes (por ejemplo, el cotilo no se colocó perfectamente en el centro del trapecio o el vástago era oblicuo con respecto a la cavidad medular del metacarpiano); (b) la posición del implante cambió levemente al principio, después se integró y continúa sin aflojamiento, en seis pacientes; (c) zonas parciales de radiolucencia bien delimita-

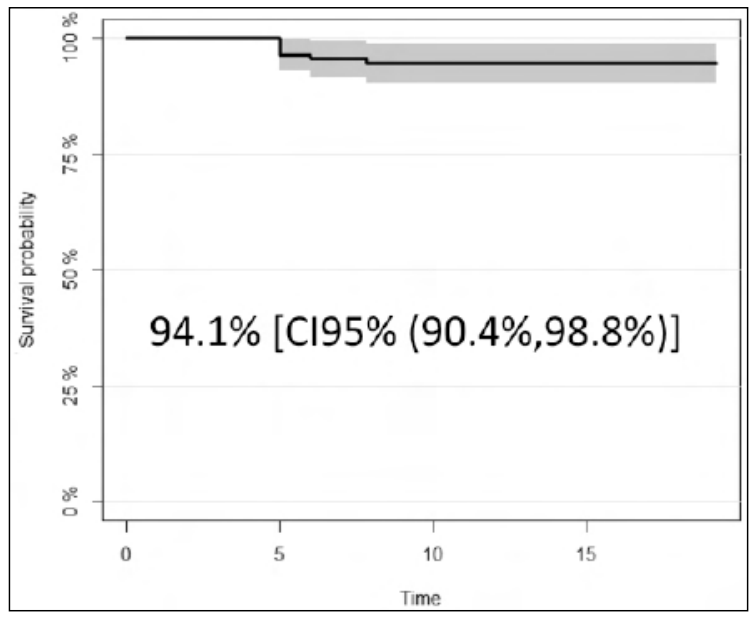

Figura 6. Curva de supervivencia de prótesis funcional de Kaplan-Meier a los 10 años de evolución. Se ha prolongado hasta los 15 años porque hay algunas prótesis revisadas con más de 10 años.

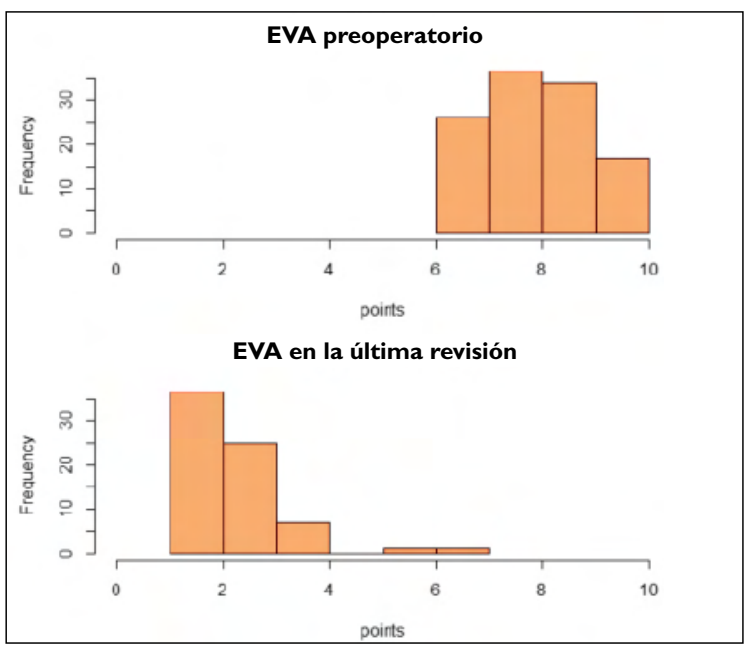

Figura 7. EVA: arriba, en el preoperatorio, abajo, en la revisión de los 10 años.

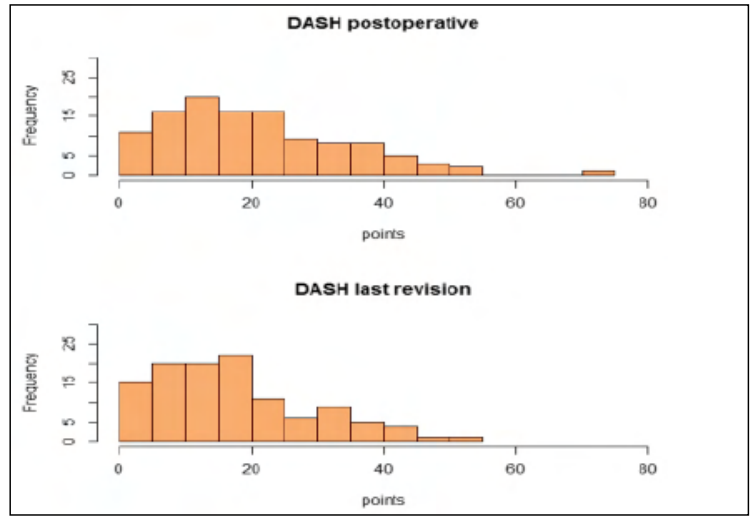

Figura 8. Puntuación en la escala DASH: arriba, en el postoperatorio a 5 años, abajo, en la revisión de los 10 años. 


\section{TABLA I - MOVILIDAD PREOPERATORIA Y EN LA REVISIÓN FINAL}

\begin{tabular}{|c|c|c|}
\hline & Preoperatorio & Post-op 10 años \\
\hline \multicolumn{3}{|l|}{ Anteposición (Abdución Palmar) } \\
\hline $0^{\circ}$ a $20^{\circ}$ & $72,3 \%$ & $10,8 \%$ \\
\hline $20^{\circ} \mathrm{a} 40^{\circ}$ & $27,7 \%$ & $89,2 \%$ \\
\hline \multicolumn{3}{|l|}{ Retroposición } \\
\hline$<5^{\circ}$ & $49,2 \%$ & $7,7 \%$ \\
\hline$>5^{\circ}$ & $50,8 \%$ & $92,3 \%$ \\
\hline \multicolumn{3}{|l|}{ Abdución (Abdución Radial) } \\
\hline$<10^{\circ}$ & $75,4 \%$ & $81,5 \%$ \\
\hline $10^{\circ}$ a $30^{\circ}$ & $24,6 \%$ & $18,5 \%$ \\
\hline \multicolumn{3}{|l|}{ Abdución } \\
\hline $0^{\circ}$ a $10^{\circ}$ & $52,3 \%$ & $10,8 \%$ \\
\hline $10^{\circ}$ a $25^{\circ}$ & $47,7 \%$ & $89,2 \%$ \\
\hline \multicolumn{3}{|l|}{ Oposición (Kapandji) } \\
\hline I a 5 & $24,6 \%$ & 0 \\
\hline 6 a 8 & $52,3 \%$ & $29,2 \%$ \\
\hline 9 y 10 & $23,1 \%$ & $70,8 \%$ \\
\hline
\end{tabular}

das alrededor de la copa que no ha cambiado desde el postoperatorio inmediato hasta el final del seguimiento, en dos pacientes; (d) calcificaciones de hueso ectópico, que han aparecido a lo largo de los años, alrededor de la base del vástago, en siete pacientes, y (e) en tres pacientes se juntan varias alteraciones: ligera mal-posición del cotilo; vástago oblicuo en sentido radial proximal-cubital distal, y laxitud del lado cubital de la MF, que provocan subluxación de la prótesis al realizar la retroposición del pulgar.

\section{RESULTADOS}

\section{Complicaciones perioperatorias:}

Las complicaciones perioperatorias incluyen: fractura sin desplazamiento del trapecio en dos pacientes; fractura oblicua proximal del metacarpiano; con leve desplazamiento, en dos pacientes; una falsa vía del vástago, que protruyó por el lado dorsal del metacarpiano; dos luxaciones tempranas de las que se hablará a continuación, y problemas de las partes blandas en once pacientes. En los pacientes con fractura del trapecio o del metacarpiano se prolongó la inmovilización postoperatoria hasta las 5 semanas y consolidaron sin problemas. La paciente con falsa vía del vástago ha pasado la revisión de los 10 años con el vástago en la misma posición, mantiene una prótesis funcional, sin signos de aflojamiento. En la revisión de los 3 meses once pacientes se quejaban de parestesias 0 disestesias moderadas del territorio radial del pulgar, pero solamente dos mostraban leves parestesias a los
10 años de evolución. Tres pacientes han presentado SDRC tipo I que se han resuelto con revisiones para apoyo psicológico frecuentes y rehabilitación. Cinco pacientes han mostrado intolerancia a los puntos 0 leves dehiscencias superficiales, que se han resuelto sin problemas. No ha existido evidencia de infección profunda en ningún paciente.

\section{Luxaciones:}

Han existido un total de seis luxaciones (5,5\%) [Cl95\% $(0.9 \%, 9.5 \%)]$ : dos tempranas y cuatro tardías.

Las tempranas (antes de los tres meses de evolución): Paciente número 3 de la serie, de 56 años de edad, ama de casa, operada de la mano izquierda, se descubrió la prótesis luxada al retirar la inmovilización postoperatoria, la paciente rechazó la revisión quirúrgica porque no presentaba muchas molestias, ha sido revisada en varias ocasiones hasta los 10 años de evolución y se la ha propuesto cirugía de revisión, que ha rechazado. La paciente número 8, de 65 años, ama de casa, operada de la mano izquierda. Se observó luxación a los 15 días de retirar la inmovilización postoperatoria. Se resolvió con revisión quirúrgica (extirpación del osteofito cubital del trapecio que provocaba el choque óseo y luxaba la prótesis por contacto, y recambio a cuello largo), la paciente se encuentra perfectamente en la revisión de los 10 años.

Las cuatro tardías (después de los tres meses): dos sin traumatismo y dos postraumáticas. Las dos sin traumatismo corresponden a la paciente número 50 , de 68 años, ama de casa, operada del lado izquierdo, con trapecio de pequeño tamaño, laxitud en hiperextensión de la articulación metacarpofalángica, el control postoperatorio mostraba ligero mal posicionamiento del trapecio, se luxó a los 7 años de evolución, sin traumatismo relevante y fue revisada quirúrgicamente mediante reducción a cielo abierto y plastia ligamentosa con la mitad del APL que se pasó alrededor del Flexor Carpi Radialis y se devolvió sobre sí mismo, suturándolo en la base del metacarpiano. En la revisión de los 10 años la prótesis estaba subluxada, tenía hiperextensión de la articulación MF y presenta un DASH de 50,3 y un EVA de 6. Se ha considerado como prótesis fallida la segunda luxación sin traumatismo corresponde a la paciente número 77 , de 54 años, auxiliar de clínica, operada del lado izquierdo, tuvo una luxación, sin traumatismo aparente. A los 3 años de evolución, se realizó en otro centro reducción y capsuloplastia, en la revisión de los 10 años la prótesis estaba luxada, se observaba laxitud en hiperextensión de la articulación MF, como la paciente no 
tenía dolor (EVA de 2 y DASH de 12,5), rechazó una nueva revisión quirúrgica.

Las dos luxaciones postraumáticas corresponden a la paciente número 82, de 45 años, operada de la mano derecha, trabajadora de la limpieza, el cotilo quedó ligeramente mal posicionado (hundimiento radial y dorsal), se luxó a los 7 años de evolución al chocar la mano contra el borde de una piscina, presenta además laxitud cubital e hiperextensión de la articulación MF. Ha sido revisada recientemente, realizando plastia con el APL y artrodesis de los dos sesamoideos, radial y cubital a la cabeza del metacarpiano para corregir la laxitud de la MF. La segunda corresponde a la paciente número 97, de 74 años, operada de la mano izquierda, con trapecio pequeño tamaño, el cotilo se colocó excesivamente desplazado hacia volar, se luxó por una caída sobre la mano a los 5 años de evolución y sigue luxada a los 10 años, ha rechazado la cirugía de revisión, presenta un EVA de 3 y DASH de 38,2.

\section{Aflojamientos de los componentes:}

Han existido en total ocho aflojamientos del cotilo protésico $(7,3 \%)$ [Cl95\% (2.1\%,1 I.9\%)] que se describen a continuación: 3 retirados, 2 recambiados y 3 que siguen colocados.

Retirada del cotilo: Los tres eran dolorosos y provocaban la subluxación o luxación de la prótesis incompatibles con la funcionalidad. La paciente número 66, de 7 I años, religiosa, operada del lado derecho, presentaba trapecio de pequeño tamaño, a los 6 años de evolución notó un dolor al apoyar con fuerza la mano contra una pared después de tropezar. Consultó por dolor y se observó aflojamiento de cotilo; se retiró el cotilo protésico y el trapecio y se realizó una tenosuspensión con el ABPL. La paciente se encuentran indolora después de 3 años de la cirugía de revisión, con EVA 2 y DASH 25. La paciente número 17, de 45 años, médico, tenía colocada una prótesis en el lado derecho el año anterior y se operó del lado izquierdo, la cirugía fue difícil por tratarse de un trapecio pequeño, y el cotilo quedó mal orientado (cubital y volar), a los 9 años presentó aflojamiento del cotilo y dolor. La paciente número 90 , de 57 años, dependienta, operada del lado derecho, con trapecio pequeño, el control radiográfico postoperatorio fue correcto. Consultó a los 8 años de evolución por dolor y se observó aflojamiento de cotilo. En estas dos últimas pacientes, solamente se retiró el cotilo protésico y se colocó Surgicel en el espacio vacío que quedaba, una de ellas (número 17) está indolora a los 4 años de la revisión quirúrgica, la otra (número 90) sigue con dolor a los dos años de evolución (se le ha ofrecido la trapecectomía completa y tenosuspensión). En las tres pacientes se ha dejado el vástago, que se encontraba completamente adherido al metacarpiano.

Recambio del cotilo: La paciente número |8, de 63 años, ama de casa, con poliartrosis y prótesis en ambas rodillas, trapecio tamaño medio, con buena posición postoperatoria del cotilo y vástago oblicuo, sufrió una caída sobre la mano a los tres años y se observó luxación y aflojamiento del cotilo. El trapecio mostraba buen capital óseo por lo que se procedió a limpieza, injertos óseos autólogos de radio distal y colocación de un nuevo cotilo protésico. A los 7 años de la cirugía de revisión, se observa el cotilo integrado con cierta malposición y subluxación de la prótesis (EVA 3 y DASH 38). La paciente número 43, de 52 años, trabajadora de la limpieza, con trapecio de tamaño medio, presentaba buena orientación postoperatoria del cotilo y vástago oblicuo, a los 9,5 años sin traumatismo previo comenzó con dolor y se observó aflojamiento del mismo. El trapecio mostraba buen capital óseo por lo que se procedió a: limpieza, injerto óseo autólogo en bloque de cresta iliaca sobre el que se colocó un nuevo cotilo protésico. A los 6 meses la paciente se encuentra indolora y el nuevo cotilo está en posición correcta y parece integrado en el injerto óseo.

\section{Aflojamientos indoloros sin alteración del posicionamiento: La paciente número 20 , de 53} años, ama de casa, operada del lado derecho, con poliartrosis, y trapecio pequeño tamaño. El control postoperatorio era aceptable, salvo oblicuidad del vástago, caminaba con un bastón en esa mano, desde los 5 años de evolución, por estar operada de artrosis de tobillo. En la revisión de los 10 años se observó aflojamiento del cotilo, sin alteraciones en la posición, con EVA 3 y DASH 25, se la ofreció revisión quirúrgica, que no aceptó. La paciente número 22, de 56 años, ama de casa, operada del lado derecho, con poliartrosis, con trapecio de tamaño medio y control postoperatorio correcto. A los 7 años presentó aflojamiento del cotilo, como consecuencia de una hipocalcemia muy severa mantenida, secundaria a la resección involuntaria de las glándulas paratiroides en una operación de bocio por hipertiroidismo, ha sido revisada en repetidas ocasiones desde los 7 a los 13 años de evolución y ha rechazado la revisión quirúrgica de la prótesis que le hemos ofrecido, en la última revisión presenta EVA 3 y DASH 28,4. La paciente número 63, de 62 años, ama de casa, con poliartrosis, 
operada del lado izquierdo, llevaba una prótesis en el lado derecho desde hacía dos años, con trapecio de pequeño tamaño, control postoperatorio correcto. Se observó aflojamiento en una revisión de la paciente a los 7 años, se la ha seguido hasta los 10 años, que presentaba EVA 3 y DASH 25. En varias ocasiones se ha propuesto a la paciente la revisión, que ha rechazado por encontrarse aceptablemente (Fig. 5).

No hemos observado aflojamientos en el vástago en ningún paciente.

\section{Hundimientos:}

La paciente número 43, de 54 años, limpiadora, operada del lado izquierdo, llevaba prótesis en el lado derecho desde hacía dos años, el control radiográfico postoperatorio fue correcto, posteriormente, en una revisión a los 9 años, la paciente tenía chasquidos en la zona de la artroplastia, que se debían a subluxación longitudinal, por hundimiento del cotilo de unos $2 \mathrm{~mm}$., junto con calcificaciones en el entorno de la parte proximal del vástago. No existía aflojamiento del cotilo en la radiografía y se corroboró en la cirugía. Se solucionó con la revisión quirúrgica: recambio del cuello medio que tenía, a un cuello largo. No hemos visto hundimiento de los componentes en el resto de los pacientes, o como mucho existe un levísimo hundimiento de adaptación del cotilo de I mm.

\section{Revisiones quirúrgicas:}

En resumen, se han realizado diez $(8,6 \%)$ revisiones quirúrgicas: cuatro por luxaciones, cinco por aflojamiento del cotilo y una por inestabilidad longitudinal. Todos los pacientes fueron operados en una ocasión, salvo la número 43 (bilateral), en la que se han revisado ambas prótesis. En cinco ocasiones se ha convertido una prótesis fallida en una prótesis funcional, en tres se han realizado retirada de la prótesis y en dos la cirugía de revisión ha sido infructuosa.

\section{Alteraciones de la articulación MF:}

En la revisión de los 10 años, dieciséis pulgares (14,7\%) mostraban laxitud en el lado cubital de la MF y en seis $(5,5 \%)$ se asociaban la laxitud cubital y laxitud en hiperextensión. Tres de ellos mostraban subluxación de la prótesis por el lado radial cuando realizaban retroposición del pulgar y dos de ellos tuvieron luxación de la prótesis. Tres de los seis pacientes con alteraciones de laxitud combinada de la articulación MF han tenido problemas de luxación de la prótesis. Por ello, ahora tratamos las laxitudes de la articulación MF en el mismo tiempo quirúrgico mediante retensado capsular y artrodesis del sesamoideo a la cabeza del metacarpiano. Además, posicionamos el cotilo protésico con ligera inclinación hacia cubital si la laxitud de la articulación MF es cubital o ligera inclinación hacia palmar si la laxitud es volar.

\section{Alteraciones de la articulación escafo- trapecio-trapezoidea (ETT):}

Doce (I \%) pacientes padecían artrosis de la ETT. Cuando existe una artrosis y la articulación es estable no hay problemas con la prótesis, y evolucionan de manera similar a cuando no existe, pero cuando hay inestabilidad, con ligera subluxación de la articulación esta se puede incrementar con el tiempo y debemos preverlo antes de colocar el implante.

\section{Factores de mal pronóstico}

Al revisar esta serie se ha visto que existen variables preoperatorias, intraoperatorias y postoperatorias que se relacionan con una peor evolución clínica y/o radiográfica.

Los factores preoperatorios son: el tamaño pequeño del trapecio, las calcificaciones periarticulares y la subluxación trapecio-metacarpiana superior a la mitad de la longitud dorso-palmar de la base del metacarpiano.

Los factores relacionados con la técnica quirúrgica son: la vía de abordaje y la experiencia del cirujano en la colocación de la prótesis.

Las postoperatorias son: la correcta inmovilización postoperatoria de 3 semanas; la existencia de poliartrosis en el enfermo que le hará caminar con ayuda de bastones y sufrir múltiples situaciones de estrés sobre la prótesis, y las explicaciones necesarias al enfermo del uso que debe hacer de dicho implante. Existen múltiples enfermos con actividad laboral importante y tareas repetitivas, que son conscientes de que llevan una prótesis, hacen un uso correcto de ella y presentan evolución favorable en la revisión de los 10 años.

Nuestro desafío es saber en modelos multivariantes qué peso tiene cada una de estas variables.

\section{DISCUSIÓN}

La artroplastia protésica trapecio-metacarpiana del pulgar ha sido considerada un proceso poco fiable porque existían pocos estudios a largo plazo que mostraran buenos resultados ${ }^{31}$. Este hecho puede ser debido a que algunas artroplastias no consiguen reproducir la anatomía de la articulación ${ }^{22,23}$, pero las 
que la reproducen, tampoco han conseguido éxito 32 . Las artroplastias tipo "ball and socket" son las que han demostrado los mejores resultados $1,3,15-21$.

Nuestra experiencia con la prótesis no cementada y no constreñida de $\mathrm{ARPE}^{\circledR}$ para el tratamiento de la artrosis trapecio-metacarpiana es buena. La tasa de supervivencia a los 10 años de los implantes que siguen siendo funcionales es del 94,1\%. Estos resultados mejoran los publicados en series de pacientes con seguimientos a largo plazo similares a los nuestros con prótesis cementadas y constreñidas de De la Caffinière $1,17,20$. Los resultados de esta serie son muy parecidos a los publicados por Allami y cols. ${ }^{33}$, en 2006, con la artroplastia de cadera (patrón oro de las prótesis).

Los resultados clínicos de los pacientes de esta serie con respecto a fuerza de la pinza, desaparición del dolor y puntuación en el DASH son mejores que los publicados en series de pacientes en los que se ha realizado trapecectomía o trapecectomía asociada a tenosuspensión, con revisiones a 6 años de evolución ${ }^{6}$. Aunque los porcentajes de fallo $(6,4 \%)$ sean ligeramente superiores a los publicados en la trapecectomía $(3,6 \%)$, dos de las prótesis que hemos considerado fallidas, presentan pulgares funcionales y están indoloras después de la revisión quirúrgica. En el resto, el 85\% estaban indoloras o con leve dolor después de ejercicio intenso, comparado con el $80 \%$ de la trapecectomía, y el $87 \%$ de la trapecectomía asociada a tenosuspensión. En la fuerza de la pinza también superan las prótesis $(5,6 \mathrm{Kg}$.) a la trapecectomía (3,7 $\mathrm{Kg}$.) y a la trapecectomía con tenosuspensión $(4, \mathrm{I} \mathrm{Kg}$.).

La mayoría de las complicaciones que hemos tenido, en lo que coincidimos con Van Capelle y $\operatorname{cols}^{34}$ se deben a un error en la indicación y/o en la técnica quirúrgica. Con respecto a la técnica quirúrgica hay que tener en cuenta que existe una curva de aprendizaje importante, ya que más de la mitad de las complicaciones graves de nuestra serie ocurrieron en las primeras 20 artroplastias.

Es precisa una técnica rigurosa y sistemática ${ }^{19}$. Nuestra experiencia en la utilización de estos implantes nos ha permitido introducir diferentes modificaciones tanto en la vía de acceso, como en la técnica quirúrgica, que mejoran la colocación y el resultado a largo plazo del posicionamiento y ajuste de los implantes.

El tamaño de la muestra y el largo seguimiento de los pacientes de este estudio permite que se puedan sacar conclusiones, aunque el diseño tenga deficiencias como el que no sea un ensayo clínico randomizado. Un solo cirujano (M-F) ha sido el responsable de tratar y evaluar a los pacientes por lo que la posibilidad de sesgo debido a la variabilidad entre observadores se puede descartar. El dolor residual y la satisfacción del paciente se han medido usando criterios objetivos: la escala EVA y la versión del cuestionario DASH validada en Castellano y completada por los propios pacientes.

Como ha sido sugerido por otros autores ${ }^{18}$, debemos poner énfasis en la necesidad de crear un registro de prótesis articulares de la articulación trapecio-metacarpiana para registrar todos los fallos de los implantes y las tasas de revisión.

\section{CONCLUSIÓN}

Este estudio demuestra que la prótesis de $\operatorname{ARPE}^{\circledR}$ (Biomet, Warsaw, Indiana) es una opción segura y fiable para el tratamiento de la artrosis trapecio-metacarpiana de los pacientes en estadio III y algunos en estadio IV de la clasificación de Eaton, con buen capital óseo, sobre todo en el trapecio y aun con demanda funcional y laboral media-alta.

\section{CONFLICTOS DE INTERESES}

Los autores declaran no tener conflictos de intereses.

\section{BIBLIOGRAFÍA}

I. Amillo S, Samper A, Illescas JA. Resultados a largo plazo de la artroplastia total de la articulación trapecio-metacarpiana mediante la prótesis cementada "de la Caffinière". Rev Med Univ Navarra. 2002,46:17-20.

2. Apard T., Saint-Cast Y. Les reprises de prothèses trapezométacarpiennes ARPE® par trapézectomie. Ligamentoplastie selon la technique de Jones: une étude homogène de six cas et revue de la littérature. Chir Main. 2007;26:95- 102.

3. Brutus J P, Kinnen L. Remplacement prothétique total trapézométacarpiernne au moyen de la prothèse ARPE dans le traitement de la rhizarthrose: notre expérience á court terme dans une série 
personnelle de 63 cas consécutifs. Chir Main. 2004;23:224-8.

4. Fischer J, Shivarathre D, Quinton D. Management of long term complications following trapeziectomy. J Bone Joint Surg Br. 20 I I;93(Supp III): 301.

5. Gangopadhyay S, McKenna H, Burke FD, Davis TRC. Five- to I 8-year follow-up for treatment of trapeziometacarpal osteoarthritis: a prospective comparison of excision, tendon interposition, and ligament reconstruction and tendon interposition. J Hand Surg Am. 20 12;37:4 I I-7.

6. Salem H, Davis TR. Six year outcome excision of the trapezium for trapeziometacarpal joint osteoarthritis: is it improved by ligament reconstruction and temporary Kirschner wire insertion? J Hand Surg Eur. 2012;37:21 I-9.

7. De la Caffinière JY. Prothèse totale trapézométcarpienne. Rev Chir Orth. 1973;59:299-308.

8. Maru M, Jettoo P,Tourret L, Jones M, Irwin L.Thumb carpometacarpal osteoarthritis: trapeziectomy versus pyrocarbon interposition implant (Pi2) arthroplasty. J Hand Surg Eur. 2012;37:617-20.

9. Nilsson A, Liljensten E, Bergström C, Sollerman C. Results from a degradable TMC joint spacer (Artelon) compared with tendon arthroplasty. J Hand Surg Am. 2005;30:380-9.

10. Burke NG,Walsh J, Kelly EP.Patient-reported outcomes after Silastic replacement of the trapezium for osteoarthritis. J Hand Surg Eur. 2012;37:263-8.

I I. Kaszap B, Daecke W, Jung M. High frequency failure of the Moje thumb carpometacarpal joint arthroplasty. J Hand Surg Eur. 2012;37:610-6.

12. Comtet JJ. ARPE prostheses. En: Simmen BR, Allieu Y, Lluch A y cols. (Eds). Hand Arthroplasties. London, :Martin Dunitz; 2000. p. 249-56.

13. Lebrun C, Sartorius C, Lafosse L, Moutet F, Carpentier E. Un nouvel implant trapezo-metacarpien. Results preliminaires a propos de 60 cas. Rhumatologie. | 996:48:9|-4.

14. Regnard PJ. Electra trapezio-metacarpal prosthesis: results of the first 100 cases. J Hand Surg Br. 2006:31:621-8.

15. Apard T, Saint Cast I. Midterm results of ARPE prosthesis for the basal thumb osteoarthritis. I Bone Joint Surg Br. 2009;9I (Supp I):96.

16. Badía A, Sambandam SN. Total joint arthroplasty in the treatment of advanced stages of thumb carpometacarpal joint osteoarthritis. J Hand Surg Am. 2006;31: 1605-13.
17. Chakrabarti AJ, Robinson AH, Gallagher P. De la Caffinière thumb carpometacarpal replacements: 93 cases at 6 to 16 years follow-up. J Hand Surg Br. 1997;22:695-8.

18. Johnston P, Getgood A, Larson D, Chojnowski AJ, Chakrabarti AJ, Chapman PG. De la Caffiniere thumb trapeziometacarpal joint arthroplasty: 1626 year follow up. J Hand Surg Eur. 20 12;37:62 I-4.

19. Simón C, Rodríguez JI, Pérez C, Martín-Ferrero M. Tratamiento de la artrosis trapecio-metacarpiana mediante artroplastia total de tipo Arpe. Modificación de la técnica. Rev Iberoamer Cir Mano. 2007;35:39-5l.

20. Sondergaard L, Konradsen L, Rechnagel K. Longterm follow-up of the cemented Caffinière prosthesis for trapeziometacarpal arthroplasty. J Hand Surg Br. 1991; 6:428-30.

21. Martín-Ferrero M. Tratamiento integral de la rizartrosis. En: Cáceres E (Ed) Cursos de Actualización 39 Congreso Nacional SECOT. Madrid: Mozart Art SL; 2002. p.101-13.

22. Hernández-Cortés P, Pajares-López M, RoblesMolina MJ, Gómez-Sánchez R, Toledo-Romero MA, De Torres-Urrea J. Two-year outcomes of Elektra prosthesis for trapeziometacarpal osteoarthritis: a longitudinal cohort study. J Hand Surg Eur. 2012;37:130-7.

23. Klahn A, Nygaard M, Gvozdenovic R, Boeckstyns ME. Elektra prosthesis for trapeziometacarpal osteoarthritis: a follow-up of 39 consecutive cases. J Hand Surg Eur. 2012;37:605-9.

24. Hendrich C, Saber U, Kirschner S, Schmitz H, Martel JM. High long-term loosening rate of conical screw cups. Acta Orthop. 2006;77:886-92.

25. International Federation of Societies for Surgery of the Hand (IFSSH). Terminology for Hand Surgery. Harcourt Health Sciences; 2001.

26. Kapandji A. Clinical test of apposition and counter-apposition of the thumb. Ann Chir Main. 1986;5:67-73.

27. Eaton RG, Litler JW. Ligament reconstruction for the painful thumb metacarpal joint. J Bone Joint Surg Am. 1973;55: 1 655-66.

28. Rosales RS, Delgado EB, Diez de la Lastra I. Evaluation of the Spanish versión of the DASH and carpal tunnel syndrome health-related quality-oflife instruments: cross-cultural adaptation process and reliability. J Hand Surg Am. 2002;27: 334-43.

29. Snedecor GW, Cochran WG. Statistical Methods, 8th ed. Ames: lowa State University Press; 1989. 
30. Kaplan EL, Meier P. Non-parametric estimation from incomplete observations. J Amer Statist Assn. |958;53:457-8|.

31. Giddins G. Editorial.J Hand Surg Eur. 20 12;37:603-4.

32. Pendse A, Nisar A, Shah SZ, Bhosale A, Freeman JV, Chakrabarti I. Surface replacement trapeziometacarpal joint arthroplasty-early results. J Hand Surg Eur. 2009;34:748-57.
33. Allami MK, Fender D, Khaw FM et al. Outcome of Charnley total hip replacement across a single health region in England. The results at ten years from a regional arthroplasty register. J Bone Joint Surg Br. 2006;88: 1293-8.

34. an Cappelle HG, Elzenga P,van Horn JR: Long-term results and loosening analysis of de la Caffiniere replacements of the trapeziometacarpal joint. J Hand Surg. 1999;24:476-482. 